\title{
An improved method to determine basic probability assignment based on fuzzy mathematics
}

\author{
Leihui Xiong, and Xiaoyan $\mathrm{Su}^{*}$
}

School of Automation Engineering, Shanghai University of Electric Power, Shanghai 200090, China

\begin{abstract}
In D-S evidence theory, the determination of the basic probability assignment function (BPA) is the first and important step. However, the generation of BPA is still a problem to be solved. Based on the concepts in fuzzy mathematics, this paper proposes an improved BPA generation method. By introducing the value of the intersection point of membership function of different targets under the same index to describe the overlap degree of targets, the assignment of unknown items is optimized on this basis. This article applies it to target recognition of robot hands. The results show that the proposed method is more reliable and more accurate.
\end{abstract}

Keywords: Multi-sensor fusion, Evidence theory, Basic probability assignment, Fuzzy, target Recognition.

\section{Introduction}

The existing intelligent systems are widely involved in the application of target recognition. However, in practical engineering, the target measured by the sensor is affected by various interference factors such as weather and noise, which makes the characteristics of the target itself not so obvious, thus resulting in the fuzziness and randomness of the output of the sensor. In addition, existing sensors may have some completely unknown information, for example, ultrasonic obstacle avoidance sensor may have detection blind area [1], etc. To sum up, the fuzziness, randomness and some unknown information of the sensor output make target recognition difficult.

In order to solve the above problems, some scholars use fuzzy number to express the output of the sensor and carry out target recognition [2-5]. The methods can express and deal with fuzziness well, and achieve good results in the application of target recognition, but there are still some shortcomings in dealing with various uncertainties and subjective cognitive uncertainties.

Dempster-Shafer's evidence theory can be considered as the generalization of probability theory [6-7]. It extends the basic event space in probability theory to the power set space of basic events, and establishes the basic probability assignment (BPA) on it. It can express uncertain information more effectively. In addition, DS evidence theory

* Corresponding author: suxiaoyan@shiep.edu.cn 
provides Dempster's combination rules, which can be integrated without prior information and satisfy some excellent characteristics such as commutative law and associative law, which makes DS evidence theory widely used in the field of multi-source information fusion. Considering that DS evidence theory can model a variety of uncertain information and effectively express and deal with subjective cognitive uncertainty, domestic and overseas scholars are committed to exploring fuzzy information processing methods based on DS evidence theory. Among them, BPA generation based on fuzzy number is the first key step. Jiang et al. [8] proposed a classical BPA generation method based on fuzzy numbers. By calculating the degree to which the measured value belongs to each target attribute, the basic reliability value of different targets is assigned, and 1 minus the value of the maximum membership function is taken as the BPA assignment value of the total set. This method can effectively reflect the membership degree of the sensor report to each target, and has certain robustness under some strong interference conditions. However, when the target characteristics are similar, the accuracy is low.

In this paper, an improved BPA fuzzy generation method is proposed, which describes the overlap degree of targets by introducing the values of the intersection points of membership functions of different targets under the same index, and on this basis, the reliability assignment of unknown items is optimized. The new method has certain physical meaning and can effectively improve the accuracy of target recognition.

\section{Dempster-Shafer evidence theory}

\subsection{The frame of discernment}

Let $\theta$ be an exhaustive set of all possible values of a variable, and the elements in $\theta$ are mutually exclusive, then $\theta$ is the frame of discernment. Let $\theta$ have $N$ elements, then the power set of $\theta$ is $P(\theta)$, the power set has $2^{N}$ elements, and each element corresponds to the proposition of a case of a variable.

$$
\begin{gathered}
\theta=\left\{H_{1}, H_{2}, \ldots, H_{n}\right\} \\
P(\theta)=\left\{\phi,\left\{H_{1}\right\},\left\{H_{2}\right\}, \ldots,\left\{H_{N}\right\},\left\{H_{1} \cup H_{2}\right\},\left\{H_{1} \cup H_{3}\right\}, \ldots, \theta\right\}
\end{gathered}
$$

\subsection{Basic probability distribution function (BPA)}

For any subset $A$ belonging to $\theta$, let it correspond to a number in the interval $[0,1]$, and satisfy:

$$
\sum_{A \in P(\theta)} m(A)=1, m(\phi)=0
$$

The function $\mathrm{m}$ is called the basic probability distribution function (BPA) on $P(\theta)$, and $m(A)$ is the basic probability number of $A$. When $A \in \theta$ and $m(A) \neq 0, A$ is called a focal element of $m$.

\subsection{Rule of evidence combination}

Let $m_{1}$ and $m_{2}$ be the two basic probability distribution functions on $P(\theta)$. Their combination (Dempster orthogonal sum) is 


$$
m(A)=\frac{\sum_{B \cap C=A} m_{1}(B) m_{2}(C)}{1-k}, k=\sum_{B \cap C=\phi} m_{1}(B) m_{2}(C)
$$

where $k$ reflects the degree of conflict between the evidence.

\section{Proposed method to determine BPA function}

\subsection{Concepts related to fuzzy numbers [9]}

Given a universe of domain $U$, then a mapping $\mu_{A}: U \rightarrow[0,1]$ from $U$ to the unit interval $[0,1]$ is called a fuzzy set on $U$ or a fuzzy subset of $U$ and is denoted as $A$. The mapping (function) $\mu_{A}(x)$ or simply $A($.$) is called a membership function of fuzzy set A$. For each $x \in U, \mu_{A}(x)$ is called the membership of the element to the fuzzy set $A$. Commonly used representations of fuzzy sets are analytic method, Zadeh notation, ordered pair method, and vector method.

\subsection{The main steps of proposed method}

STEP1 In general, the sensor will accumulate certain sample data for the measured object during the long-term operation, which is helpful to the construction of fuzzy membership function. The first step is to generate fuzzy membership function based on sample data (or parameters). Commonly used fuzzy membership functions include triangle, trapezoid, normal distribution, Gaussian distribution, etc. The specific selection of fuzzy membership should be determined by combining engineering practice and available data. The selected fuzzy membership degree must be able to reflect the characteristics of the target attributes and be easy for subsequent data processing. For example, the mean value of the quality of target A after several measurements is 243 and the deviation is 35 . Normal fuzzy number membership function (Equation (5)) and triangular fuzzy number membership function (Equation (6)) can be used for modeling respectively.

$$
\begin{aligned}
& \mu_{A}(x)=e^{-\frac{(x-243)^{2}}{35^{2}}} \\
& \mu_{\mathrm{A}}(x)=\left\{\begin{array}{l}
1-\frac{|x-243|}{2 \times 35},|x-243|<70 \\
0,|x-243|>70
\end{array}\right.
\end{aligned}
$$

See Figure 1(a), (b) for graphic representation.

STEP2 Assume that the target to be recognized by the system is $H_{1}, H_{2}, \ldots, H_{m}$, that is, the identification framework is $\Theta=\left\{H_{1}, H_{2}, \ldots, H_{m}\right\}$. The sensor observes $n$ attribute features of the target, and obtains $n$ dimension characteristic measurement vector $Z=\left\{Z_{1}, Z_{2}, \ldots, Z_{n}\right\}^{T}$. Where, $Z_{i}$ represents the measured value of the $i$ th attribute. The fuzzy membership function of object $j$ under attribute $i$ is $\mu_{i j}$. Let the observed value of the attribute feature be $Z_{i}$, then $\mu_{i j}\left(Z_{i}\right)$ represents the degree to which the observed result is subordinate to target $j$ under attribute $i$.

STEP3 For the same attribute $i$, the maximum value of $\mu_{i j}\left(Z_{i}\right)$ is denoted as $U_{n}$ : 


$$
U_{n}=\max _{\mathrm{j}=1, \ldots, m} \mu_{i j}\left(Z_{\mathrm{i}}\right)
$$

To some extent, this maximum value reflects the upper boundary of determining information, and uncertainty can be expressed with $1-U_{n}$. In Jiang et al.'s method [8], $1-U_{n}$ is assigned to the universal set to express uncertainty, which is reasonable to a certain extent. However, when the target properties are similar, the recognition accuracy is low. For this reason, this paper takes into account the relationship of membership functions of different targets under the same attribute, and denoted the maximum intersection point of each membership function image as $P_{\text {max }}$ (as shown in Figure 2). The larger $P_{\max }$ (the greater degree of overlap) indicates that the target to be recognized is closer in nature. At this point, in order to make better use of the existing information, the reliability of the unknown items (i.e., the total set) should be correspondingly reduced. In this paper, $1-P_{\max }$ is used as the correction factor for the reliability assignment of unknown items. When there is a good degree of discrimination between objects, $P_{\max }$ is small, and the reliability assignment of the whole set mainly depends on $1-U_{n}$. When the target properties are close to each other, the value of $P_{\max }$ is larger, and the reliability assigned to the total set is correspondingly reduced, which is conducive to better use of the existing information. To sum up, the reliability assigned to the universal set is determined by the following formula:

$$
Q=\left(1-U_{n}\right)\left(1-P_{\max }\right)
$$

STEP4 From the above steps, we can get the trust assigned to each target under the same attribute and the trust assigned to the unknown (i.e., the total set), and normalize these to get the final BPA. An example is given to illustrate the proposed BPA generation process. Suppose that an intelligent system needs to identify two targets, $O_{1}$ and $O_{2}$. The mean and deviation of the target $O_{1}$ mass are $(243,35)$, and the mean and deviation of the target $O_{2}$ mass are $(270,60)$. Sensor $S_{1}$ is used to measure the mass attribute $m$ of the target to be recognized, and the obtained observation value is 300 . According to the above steps, the process of generating BPA is as follows:

(1) Generate normal distribution membership function based on parameters of target $O_{1}$ and $O_{2}$ (as shown in Figure 2).

(2) Under the quality attribute, the degree of membership of the observed value to the target $O_{1}$ and $O_{2}$ is obtained respectively: $\mu_{m o_{1}}\left(Z_{m}\right)=0.0705 \quad \mu_{m o_{2}}\left(Z_{m}\right)=0.7788$

(3) The level of trust assigned to the full set is: $Q=(1-0.7788)(1-0.922)=0.0173$

(4) When the measured value is normalized to 300 , the final BPA is obtained as follows:

$$
\begin{aligned}
& m_{1}\left(O_{1}\right)=\frac{0.0705}{0.0705+0.7788+0.2212 \times 0078}=0.0813 \\
& m_{1}\left(O_{2}\right)=\frac{0.7788}{0.0705+0.7788+0.2212 \times 0078}=0.8987 \\
& m_{1}(\Theta)=\frac{0.2212 \times 0.078}{0.0705+0.7788+0.2212 \times 0078}=0.0199
\end{aligned}
$$




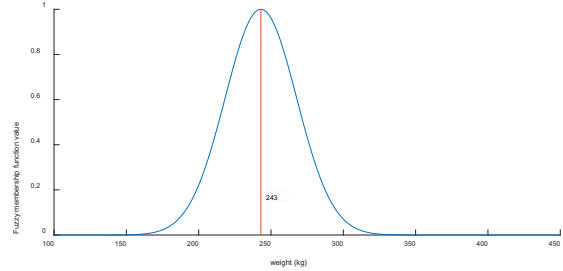

(a) Normal fuzzy number

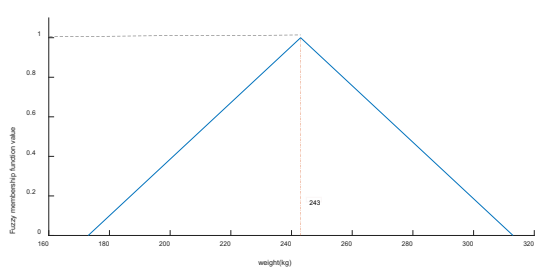

(b) Triangular fuzzy number

Fig. 1. Membership function of fuzzy number.

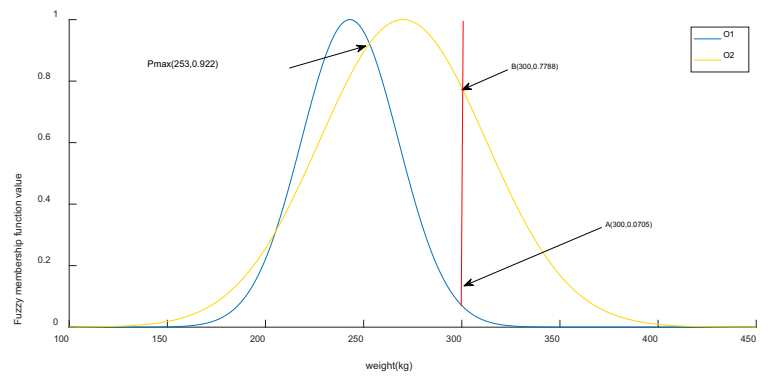

Fig. 2. Example.

\section{Application of workpiece identification}

In this paper, an example in [2] is used to illustrate the effectiveness of the proposed method. There is a robot system for classification and workpiece identification. It is assumed that there are four types of artifacts to be identified: A, B, C and D (see Table 1 for the standard values of index characteristics and their standard variances of the models of the four types of artifacts). Among them, the characteristic indexes include the shape factor $\theta_{1}$, the central moment of the section $\theta_{2}$, the surface reflection ability $\theta_{3}$, the surface roughness $\theta_{4}, \sigma_{1 j}, \sigma_{2 j}, \sigma_{3 j}$ and $\sigma_{4 j}$, respectively, which are the variances of these four characteristics.

The computer receives the data collected by the sensor and obtains the characteristic observation value vector $Z$ of the four attributes after a series of processing. $Z_{i}$ is taken to represent the measured value of attribute $i$, and then $Z=\left\{Z_{1}, Z_{2}, Z_{3}, Z_{4}\right\}^{T}$ is obtained. The actual characteristic observation vector is: $Z=(2.15,2.30,2.80,2.12)^{T}$

Table 1. The index characteristic standard value of the workpiece model and its standard variance.

\begin{tabular}{ccccccccc}
\hline Feature & $\overline{\theta_{1}}$ & $\overline{\sigma_{1}}$ & $\overline{\theta_{2}}$ & $\overline{\sigma_{2}}$ & $\overline{\theta_{3}}$ & $\overline{\sigma_{3}}$ & $\overline{\theta_{4}}$ & $\overline{\sigma_{4}}$ \\
\hline objective & 1.30 & 0.12 & 1.86 & 0.10 & 3.07 & 0.11 & 2.75 & 0.25 \\
$\mathrm{~A}$ & 2.43 & 0.37 & 3.71 & 0.17 & 2.28 & 0.37 & 2.34 & 0.07 \\
$\mathrm{~B}$ & 2.18 & 0.15 & 1.93 & 0.11 & 1.37 & 0.13 & 1.52 & 0.12 \\
$\mathrm{D}$ & 1.85 & 0.19 & 2.52 & 0.23 & 2.97 & 0.25 & 1.93 & 0.19 \\
\hline
\end{tabular}

In combination with the practical problems and considering the principle of convenient processing in the future, this paper chooses triangular fuzzy number to describe the characteristics of the object. Here, the fuzzy membership function corresponding to the workpiece type $j$ under attribute $i$ is denoted as $\mu_{i j}$. 
Firstly, the triangular fuzzy membership function is determined. For example, the membership function of the shape factor of the workpiece type A can be used as follow.

$$
\mu_{\theta_{1} \mathrm{~A}}(z)=\left\{\begin{array}{l}
1-\frac{\left|z_{1}-1.30\right|}{2 \times 0.12},\left|z_{1}-1.30\right|<0.24 \\
0,\left|z_{1}-1.30\right|>0.24
\end{array}\right.
$$

In the same way, membership function of characteristic indexes of other targets can also be expressed.

According to Step 2, the characteristic observed value vector $Z=(2.15,2.30,2.80,2.12)^{T}$ was substituted into the membership function accordingly. The results are shown in Table 2:

Table 2. The membership function results in the example.

\begin{tabular}{ccccc}
\hline Objective & $\mu_{\theta, j}\left(Z_{1}\right)$ & $\mu_{\theta_{2 j}}\left(Z_{2}\right)$ & $\mu_{\theta_{j}}\left(Z_{3}\right)$ & $\mu_{o, j}\left(Z_{4}\right)$ \\
\hline A & 0 & 0 & 0 & 0 \\
B & 0.6216 & 0 & 0.2973 & 0 \\
C & 0.9000 & 0 & 0 & 0 \\
D & 0.2105 & 0.5217 & 0.6600 & 0.5000 \\
\hline
\end{tabular}

Then calculate the trust degree assigned to the full set, and get the value of $P_{\max }$ according to Step 3 as shown in Table 3.

Table 3. The results of the $P_{\max }$.

\begin{tabular}{ccccc}
\hline Attribute & $\theta_{1}$ & $\theta_{2}$ & $\theta_{3}$ & $\theta_{4}$ \\
\hline$P_{\max }$ & $0.7 r_{0} 00$ & $0.83 \gamma_{4}$ & 0.8608 & 0.3600 \\
\hline
\end{tabular}

Thus, the reliability value assigned to the total set is obtained according to Equation (8), as shown in Table 4.

Table 4. The reliability value assigned to the universal set.

\begin{tabular}{cllll}
\hline Attribute & $\theta_{1}$ & $\theta_{2}$ & $\theta_{3}$ & $\theta_{4}$ \\
\hline$\left(1-U_{n}\right)\left(1-P_{\max }\right)$ & 0.0240 & 0.0783 & 0.0473 & 0.3200 \\
\hline
\end{tabular}

Normalize the above results according to Step 4, and the BPA generated is as follows:

$$
\begin{aligned}
& 1, \mathrm{~m}_{1}(\mathrm{~A})=0 ; \mathrm{m}_{1}(\mathrm{~B})=0.3540 ; \mathrm{m}_{1}(\mathrm{C})=0.5125 ; \mathrm{m}_{1}(\mathrm{D})=0.1199 ; \mathrm{m}_{1}(\mathrm{Q})=0.0137 \\
& 2, \mathrm{~m}_{2}(\mathrm{~A})=0 ; \mathrm{m}_{2}(B)=0 ; \mathrm{m}_{2}(\mathrm{C})=0 ; \mathrm{m}_{2}(\mathrm{D})=0.8696 ; \mathrm{m}_{2}(\mathrm{Q})=0.13043 \\
& 3, \mathrm{~m}_{3}(\mathrm{~A})=0 ; \mathrm{m}_{3}(\mathrm{~B})=0.2959 ; \mathrm{m}_{3}(\mathrm{C})=0 ; \mathrm{m}_{3}(\mathrm{D})=0.6570 ; \mathrm{m}_{3}(\mathrm{Q})=0.04708 \\
& 4, \mathrm{~m}_{4}(\mathrm{~A})=0 ; \mathrm{m}_{4}(B)=0 ; \mathrm{m}_{4}(\mathrm{C})=0 ; \mathrm{m}_{4}(\mathrm{D})=0.609756 ; \mathrm{m}_{4}(\mathrm{Q})=0.3902
\end{aligned}
$$

Then, according to Dempster's combination rule (see Equation (4)), the fusion results of this method are calculated as shown in Table 5.

Table 5. The fusion results.

\begin{tabular}{cccccc}
\hline Sensor Fusion & & $m_{1}$ & $m_{1} \oplus m_{2}$ & $m_{1} \oplus m_{2} \oplus m_{3}$ & $m_{1} \oplus m_{2} \oplus m_{3} \oplus m_{4}$ \\
\hline \multirow{3}{*}{ The fusion } & $\mathrm{m}(\mathrm{A})$ & 0.0000 & 0.0000 & 0.0000 & 0.0000 \\
results & $\mathrm{m}(\mathrm{B})$ & 0.5340 & 0.1873 & 0.1441 & 0.0630 \\
& $\mathrm{~m}(\mathrm{C})$ & 0.5125 & 0.2711 & 0.0277 & 0.0121 \\
& $\mathrm{~m}(\mathrm{D})$ & 0.1199 & 0.5344 & 0.8274 & 0.9278 \\
& $\mathrm{~m}(\Theta)$ & 0.0137 & 0.0072 & $8.6630 \times 10^{4}$ & $8.6630 \times 10^{-4}$ \\
\hline
\end{tabular}

As can be seen from Table 5, with the integration of evidence, the degree of support for artifact type D gradually increases, and the reliability assigned to the unknown gradually decreases. 
The method in this paper was compared with the Chen et al.'s method [2] and Jiang et al.'s method [8], and the results are shown in Table 6.

Table 6. The synthesis results of different methods.

\begin{tabular}{cccccc}
\hline Method & $m(A)$ & $m(B)$ & $m(C)$ & $m(D)$ & $m(\Theta)$ \\
\hline Chen et al.'s method[2] & 0.1596 & 0.2534 & 0.2425 & 0.3445 & 0 \\
Jang et al.'s method[20] & 0 & 0.2098 & 0.1507 & 0.6228 & 0.0168 \\
Proposed method & 0 & 0.0630 & 0.0121 & 0.9278 & $8.6630 \times 10^{-4}$ \\
\hline
\end{tabular}

It can be seen from Table 6 that the three methods all have the greatest support for type $\mathrm{D}$ and can correctly identify the artifacts belonging to type D. By comparing the three methods, it can be seen that the method in this paper is significantly more supportive to D than the other two methods. This helps to get the correct results in complex situations and improve the identification accuracy of the intelligent system. The method in this paper assigns part of the basic probability to the unknown term $\Theta$, so that the method has certain robustness under the action of strong disturbance. At the same time, this method also optimizes the reliability assignment of the unknown item $\Theta$ based on the overlap degree of membership function, which can improve the accuracy of target recognition to a large extent.

\section{Conclusion}

This paper presents an improved fuzzy generation method for BPA. This method can effectively express the membership degree of the measured value of the sensor to each recognition target, and judge the proximity degree of the target property based on the overlap degree of fuzzy membership function of different workpiece types under the same index. The information of proximity is used to optimize the reliability assignment of unknown items and improve the system identification accuracy. An example of intelligent robot workpiece recognition is given to illustrate the effectiveness of the method.

This research was funded by the Shanghai Natural Science Foundation (Grant No. 19ZR1420700), the "Chenguang Program" supported by the Shanghai Education Development Foundation and the Shanghai Municipal Education 275 Commission (Grant No. 16CG60), Shanghai Key Laboratory of Power Station Automation Technology (Grant No. 13DZ2273800).

\section{References}

1. Zaibin Jiao,Rundong Wu. A New Method to Improve Fault Location Accuracy in Transmission Line Based on Fuzzy Multi-Sensor Data Fusion[J]. IEEE Transactions on Smart Grid ,2019,10(4).

2. Chen Shouyu, Hu Jimin. Variable Fuzzy Method and Its Application in Workpiece Recognition [J]. Systems Engineering and Electronics,2006(09):1325-1328. (in Chinese)

3. Xia Zhang, Youchao Sun, Zhifan Qiu, Junping Bao, Yanjun Zhang. Adaptive NeuroFuzzy Fusion of Multi-Sensor Data for Monitoring a Pilot's Workload Condition[J]. Sensors, 2019,19(16).

4. Xinbin Zhou, Tong Peng. Application of multi-sensor fuzzy information fusion algorithm in industrial safety monitoring system[J]. Safety Science,2020,122.

5. Zaibin Jiao, Rundong Wu. A New Method to Improve Fault Location Accuracy in Transmission Line Based on Fuzzy Multi-Sensor Data Fusion[J]. IEEE Transactions on Smart Grid ,2019,10(4). 
6. A P Dempster Upper and Lower Probabilities Induced by a Multivalued Mapping[J]. The Annals of Mathematical Statistics,1967,38(2).

7. Shafer Glenn. A Mathematical Theory of Evidence[M]. New Jersey: Princeton University Press:2020-06-30.

8. Jiang Wen, Zhang An, Yang Qi. A Fuzzy Generation of Basic Probability Assignment and Its Application in Data Fusion[J]. Chinese Journal of Sensors and Actuators,2008(10):1717-1720. (in Chinese)

9. Zhang Xuewei,Li Hanshan. Research on Transformer Fault Diagnosis Method and Calculation Model by using Fuzzy Data Fusion in Multi sensor Detection System[J]. Optik,2018,176. 